\title{
The p.q.-Baer Property of Fixed Rings under Finite Group Action
}

\author{
Ling Jin, Hailan Jin \\ Department of Mathematics, College of Sciences, Yanbian University, Yanji, China \\ Email: "hljin98@ybu.edu.cn, hljin98@hanmail.net
}

Received August 21, 2012; revised September 30, 2012; accepted October 8, 2012

\begin{abstract}
A ring $R$ is called right principally quasi-Baer (simply, right p.q.-Baer) if the right annihilator of every principal right ideal of $R$ is generated by an idempotent. For a ring $R$, let $G$ be a finite group of ring automorphisms of $R$. We denote the fixed ring of $R$ under $G$ by $R^{G}$. In this work, we investigated the right p.q.-Baer property of fixed rings under finite group action. Assume that $R$ is a semiprime ring with a finite group $G$ of $\mathrm{X}$-outer ring automorphisms of $R$. Then we show that: 1) If $R$ is $G$-p.q.-Baer, then $R^{G}$ is p.q.-Baer; 2) If $R$ is p.q.-Baer, then $R^{G}$ are p.q.-Baer.
\end{abstract}

Keywords: p.q.-Baer Property; Fixed Ring; Group Action

\section{Introduction}

Throughout this paper all rings are associative with identity. Recall from [1] that a ring $R$ is called right principally quasi-Baer (simply, right p.q.-Baer) if the right annihilator of every principal right ideal of $R$ is generated, as a right ideal, by an idempotent of $R$. A left principally quasi-Baer (simply, left p.q.-Baer) ring is defined similarly. Right p.q.-Baer rings have been initially studied in [1]. For more details on (right) p.q.-Baer rings, see [1-6].

Recall from [7] (see also [8]) that a ring $R$ is called quasi-Baer if the right annihilator of every right ideal is generated, as a right ideal, by an idempotent of $R$. A ring $R$ is called biregular if for each $x \in R, R x R=e R$ for some central idempotent $e \in R$. We note that the class of right p.q.-Baer rings is a generalization of the classes of quasi-Baer rings and biregular rings.

For a ring $R$, we use $Q(R)$ to denote a fixed maximal right ring of quotients of $R$. According to [9] an idempotent $e$ of a ring $R$ is called left (resp., right) semicentral if $a e=e a e$ (resp., ea=eae ) for all $a \in R$. Equivalently, an idempotent $e$ is left (resp., right) semicentral if and only if $e R$ (resp., $R e$ ) is a two-sided ideal of $R$. For a ring $R$, we let $S_{l}(R)$ (resp., $S_{l}(R)$ ) denote the set of all left (resp., right) semicentral idempotents. An idempotent $e$ of a ring $R$ is called semicentral reduced if $S_{l}(e R e)=\{0, e\}$. Recall from [2] that a ring $R$ is called semicentral reduced if $S_{l}(R)=\{0,1\}$, i.e., 1 is a semicentral reduced idempotent of $R$.

For a nonempty subset $X$ of a ring $R$, we use $r_{R}(X)$ and $l_{R}(X)$ to denote the right annihilator and the left

"Corresponding author. annihilator of $X$ in $R$, respectively. If $R$ is a semiprime ring and $I$ is a two-sided ideal of $R$, then $r_{R}(I)=l_{R}(I)$. For a right $R$-module $M$ and a submodule $N$ of $M$, we use $N_{R} \leq^{\text {ess }} M_{R}$ and $N_{R} \leq^{\text {den }} M_{R}$ to denote that $N_{R}$ is essential in $M_{R}$ and $N_{R}$ is dense in $M_{R}$, respectively.

For a ring $R$, we let $\operatorname{Aut}(R)$ denote the group of ring automorphisms of $R$. Let $G$ be a subgroup of $\operatorname{Aut}(R)$. For $r \in R$ and $g \in G$, we let $r^{g}$ denote the image of $r$ under $g$. We use $R^{G}$ to denote the fixed ring of $R$ under $G$, that is $R^{G}=\left\{r \in R \mid r^{g}=r\right.$ for every $\left.g \in G\right\}$.

We begin with the following example.

\section{Preliminary}

Example 2.1. There exist a ring $R$ and a finite group $G$ of ring automorphisms of $R$ such that $R$ is right p.q.-Baer but $R^{G}$ is not right p.q.-Baer. Let $R=\left[\begin{array}{cc}F & F \\ 0 & F\end{array}\right]$ with a field $F$ of characteristic 2 . Then $R$ is right p.q.-Baer. Define $g \in \operatorname{Aut}(R)$ by

$$
g\left(\left[\begin{array}{ll}
a & b \\
0 & c
\end{array}\right]\right)=\left[\begin{array}{ll}
1 & 1 \\
0 & 1
\end{array}\right]^{-1}\left[\begin{array}{ll}
a & b \\
0 & c
\end{array}\right]\left[\begin{array}{ll}
1 & 1 \\
0 & 1
\end{array}\right]
$$

Then $g^{2}=1$ since the characteristic of $F$ is 2 .

Now we show that $R^{G}$ is not right p.q.-Baer. The fixed ring under $G$ is

$$
R^{G}=\left\{\left[\begin{array}{cc}
x & y \\
0 & x
\end{array}\right] \in R \mid x, y \in F\right\}
$$

By computation we see that the idempotents of $R^{G}$ are only 0 and 1 , thus $R^{G}$ is semicentral reduced. So if $R^{G}$ is 
right p.q.-Baer, then $R^{G}$ is a prime ring by [2, Lemma 4.2], a contradiction. Thus $R^{G}$ is not right p.q.-Baer.Also we can see that $R^{G}$ is not left p.q.-Baer.

Definition 2.2. Let $R$ be a semiprime ring. For $g \in \operatorname{Aut}(R)$, let

$$
\phi_{g}=\left\{x \in Q_{m}(R) \mid x r^{g}=r x \text { for each } r \in R\right\},
$$

where $Q_{m}(R)$ is the Martindale right ring of quotients of $R$ (see [10] for more on $Q_{m}(R)$ ). We say that $g$ is Xouter if $\phi_{g}=0$. A subgroup $G$ of $\operatorname{Aut}(R)$ is called Xouter on $R$ if every $1 \neq g \in G$ is X-outer. Assume that $R$ is a semiprime ring, then for $g \in \operatorname{Aut}(R)$, let

$$
\Phi_{g}=\left\{x \in Q_{m}(R) \mid x r^{g}=r x \text { for each } r \in R\right\} .
$$

For $g \in \operatorname{Aut}(R)$, we claim that $\Phi_{g}=\phi_{g}$. Obviously $\phi_{g} \subseteq \Phi_{g}$. Conversely, if $x \in \Phi_{g}$ then $x R=R x$. There exists $I_{R} \leq^{\text {den }} R_{R}$ such that $x I \subseteq R$. Therefore $R I \triangleleft R$, $(R I)_{R} \leq^{\text {den }} R_{R}$, and $x R I=R x I \subseteq R$. Thus $x \in Q_{m}(R)$, hence $x \in \phi_{g}$. Therefore $\Phi_{g}=\phi_{g}$. So if $G$ is X-outer on $R$, then $G$ can be considered as a group of ring automorph-ismms of $Q(R)$ and $G$ is X-outer on $Q(R)$. For more details for $\mathrm{X}$-outer ring automorphisms of a ring, etc., see [10, p. 396] and [11].

We say that a ring $R$ has no nonzero $n$-torsion ( $n$ is a positive integer) if $n a=0$ with $a \in R$ implies $a=0$.

Lemma 2.3. [12,13]

Let $R$ be a semiprime ring and $G$ a group of ring automorphisms of $R$. If $R^{*} G$ is semiprime, then $R^{G}$ is semiprime.

For a ring $R$, we use $\operatorname{Cen}(R)$ to denote the center of $R$.

Lemma 2.4. For a semiprime ring $R$, let $G$ be a group of X-outer ring automorphisms of $R$.

Then $\operatorname{Cen}\left(R^{*} G\right)=\operatorname{Cen}\left(R^{G}\right)$.

\section{Proof.}

Let $\alpha=a_{1} 1+a_{2} g_{2}+\cdots+a_{n} g_{n} \in \operatorname{Cen}(R)$ with $a_{i} \in R$, 1 the identity of $G$, and $g_{i} \in G$.

The $\left(a_{1} 1+a_{2} g_{2}+\cdots+a_{n} g_{n}\right) b=b\left(a_{1} 1+a_{2} g_{2}+\cdots+a_{n} g_{n}\right)$ for all $b \in R$. So $a_{1} b=b a_{1}, a_{2} b^{g_{2}}=b a_{2}, \cdots, a_{n} b^{g_{n}}=b a_{n}$ for all $b \in R$. Since $G$ is X-outer, it follows that

$a_{2}=\cdots=a_{n}=0$. Hence $\alpha=a_{1} 1=a_{1} \in R$. Also since $\alpha b=b \alpha$ for all $b \in R$, we have that $a_{1} \in \operatorname{Cen}(R)$. Note that for all $g \in G, a_{1} g=g a_{1}=a_{1}^{g^{-1}} g$ implies $a_{1}=a_{1}^{g^{-1}}$. So $\alpha=a_{1} \in \operatorname{Cen}(R)^{G}$. Thus $\operatorname{Cen}\left(R^{*} G\right)=\operatorname{Cen}(R)^{G}$.

Conversely, $\operatorname{Cen}(R)^{G} \subseteq \operatorname{Cen}\left(R^{*} G\right)$ is clear.

Therefore $\operatorname{Cen}\left(R^{*} G\right)=\operatorname{Cen}\left(R^{G}\right)$.

Lemma 2.5. [14] Assume that $R$ is a semiprime ring and $G$ is a finite group of X-outer ring automorphisms of $R$. Then $\operatorname{Cen}\left(Q(R)^{G}\right)=[\operatorname{Cen}(Q(R))]^{G}$.

Lemma 2.6. Assume that $R$ is a semiprime ring and $e \in B(Q(R))$. Let $I$ be a two-sided ideal of $R$ such that $I_{R} \leq^{\text {ess }} e R_{R}$ and $r_{R}(I)=f R$ with $f \in B(R)$. Then $e=1-f$.

Proof. Since $R$ is semiprime, $I_{R} \leq^{\text {ess }} l_{R}\left(r_{R}(I)\right)_{R}=(1-f) R$. Thus $I_{R} \leq^{\text {ess }}(1-f) Q(R)_{R}$. As $I_{R}$ ess $^{\text {ess }} e R_{R}, I_{R}$ ess $^{\text {ess }} e Q(R)_{R}$. We note that $e$ and $1-f$ are in $B(Q(R))$. So we have that $e=1-f$.

Lemma 2.7. [15] Let $R$ be a semiprime ring with a finite group $G$ of X-outer ring automorphisms of $R$.

1) For $q \in Q(R)^{G}$, let $I$ be a dense right ideal $I$ of $R^{G}$ such that $q I \subseteq R^{G}$. Then $I R$ is a dense right ideal of $R$ and the map $\tilde{q}: I R \rightarrow R$ defined by

$\tilde{q}\left(\sum a_{i} r_{i}\right)=\sum\left(a_{i}\right) r_{i}$, with $a_{i} \in I$ and $r_{i} \in R$, is a right $R$-homomorphism. Moreover $\tilde{q} \in Q(R)^{G}$.

2) The map $\sigma: Q\left(R^{G}\right) \rightarrow Q(R)^{G}$ defined by $\sigma(q)=\tilde{q}$ is a ring isomorphism.

3) Let $\tilde{q} \in Q(R)$ and $K$ a dense right ideal of $R$ such that $\tilde{q} K \subseteq R$. Then $K \cap R^{G}$ is a dense right ideal of $R^{G}$ and $\left.\tilde{q}\right|_{R^{G}}\left(K \cap R^{G}\right) \subseteq R^{G}$, where $\left.\tilde{q}\right|_{R^{G}}$ is the restriction of $\tilde{q}$ to $R^{G}$. Thus $\left.\tilde{q}\right|_{R^{G}} \in Q\left(R^{G}\right)$.

For a ring $R$ with a group $G$ of ring automorphism of $R$, we say that a right ideal $I$ of $R$ is $G$-invariant if $I^{g} \subseteq I$ for every $g \in G$, where $I^{g}=\left\{a^{g} \mid a \in I\right\}$.

Proposition 2.8. [1] Let $R$ be a semiprime ring. Then the followings are equivalent.

1) $R$ is right p.q.-Baer;

2) Every principal two-sided ideal of $R$ is right essential in a ring direct summand of $R$;

3) Every finitely generated two-sided ideal of $R$ is right essential in a ring direct summand of $R$;

4) Every principal two-sided ideal of $R$ that is closed as a right ideal is a direct summand of $R$;

5) For every principal two-sided ideal $I$ of $R, r_{R}(I)$ is right essential in a direct summand of $R$;

6) $R$ is left p.q.-Baer.

For a ring $R$ with a group $G$ of ring automorphisms of $R$, we say that a right ideal $I$ of $R$ is $G$-invariant if $I^{g} \subseteq I$ for every $g \in G$, where $I^{g}=\left\{a^{g} \mid a \in I\right\}$. Assume that $R$ is a semiprime ring with a group $G$ of ring automorphisms of $R$. We say that $R$ is $G$-p.q.-Baer if the right annihilator of every finitely generated $G$-invariant two-sided ideal is generated by an idempotent, as a right ideal. By Proposition 8, if a ring $R$ is semiprime p.q.Baer with a group $G$ of ring automorphisms of $R$, then $R$ is $G$-p.q.-Baer.

A ring $R$ is called right Rickart if the right annihilator of each element is generated by an idempotent of $R$. A left Rickart ring is defined similarly. A ring $R$ is called Rickart if $R$ is both right and left Rickart. A ring $R$ is said to be reduced if $R$ has no nonzero nilpotent element. We note that reduced Rickart rings are p.q.-Baer rings. 
We put

$$
\begin{aligned}
B_{p}(Q(R))= & \{e \in B(Q(R)) \mid \text { there exists } x \in R \text { with } \\
& \left.R \times R_{R} \leq^{\text {ess }} e R_{R}\right\} .
\end{aligned}
$$

Let $\hat{Q}_{p q \mathrm{~B}}(R)$ be the subring of $Q(R)$ generated by $R$ and $B_{p}(Q(R))$.

Lemma 2.9. [16] Assume that $R$ is a semiprime ring. Then:

1) The ring $\hat{Q}_{p q \mathrm{~B}}(R)$ is the smallest right ring of quotients of $R$ which is p.q.-Baer;

2) $R$ is p.q.-Baer if and only if $B_{p}(Q(R)) \subseteq R$.

With these preparations, in spite of Example 1, we have the following result for p.q.-Baer property of $R^{G}$ on a semiprime ring $R$ for the case when $G$ is finite and $\mathrm{X}$-outer.

\section{Main Results}

Theorem 3.1. Let $R$ be a semiprime ring with a finite group $G$ of X-outer ring automorphisms of $R$. Then:

1) If $R$ is $G$-p.q.-Baer, then $R^{G}$ is p.q.-Baer.

2) If $R$ is reduced $G$-p.q.-Baer, then $R^{G}$ is Rickart.

Proof. 1) Assume that $R$ is $G$-p.q.-Baer. To show that $R^{G}$ is p.q.-Baer, it is enough to see that $B_{p}\left(Q\left(R^{G}\right)\right) \subseteq R^{G}$ by Lemma 9 since $R^{G}$ is semiprime from Lemma 3. Let $e \in B_{p}\left(Q\left(R^{G}\right)\right)$. Then $e \in B\left(Q\left(R^{G}\right)\right)$, so $\tilde{e} \in B\left(Q\left(R^{G}\right)\right)$ by Lemma 7. From Lemma 9, there exists $a \in R^{G}$ such that $R^{G} a R_{R^{G}}^{G} \leq{ }^{\text {ess }} e R_{R^{G}}^{G}$ because $e \in B_{p}\left(Q\left(R^{G}\right)\right)$. Note that $R^{G} a R_{R^{G}}^{G} \leq \tilde{e} R_{R^{G}}^{G}$.

We show that $R^{G} a R_{R^{G}}^{G} \leq^{\text {ess }} \tilde{e} R_{R^{G}}^{G}$. To see this, say $0 \neq \tilde{e} r \in \tilde{e} R^{G}$ with $r \in R^{G}$. Then $0 \neq e r \in e R^{G}$. So there exists $b \in R^{G}$ such that $0 \neq e r b \in R^{G} a R^{G}$. Hence $0 \neq \tilde{e} r b \in R^{G} a R^{G}$.

Observe that $\left[R^{G} a R^{G} \oplus r_{R^{G}}\left(R^{G} a R^{G}\right)\right]_{R^{G}} \leq^{\text {ess }} R_{R}^{G}$, as $R^{G}$ is semiprime from Lemma 3. So

$R^{G} a R^{G} \oplus r_{R^{G}}\left(R^{G} a R^{G}\right)$ is a dense right ideal of $R^{G}$ since $R^{G}$ is semiprime. By Lemma 7, $\left[R^{G} a R^{G} \oplus r_{R^{G}}\left(R^{G} a R^{G}\right)\right] R$ is a dense right ideal of $R$. So it is essential in $R_{R}$. Hence

$$
\left[R^{G} a R^{G}+r_{R^{G}}\left(R^{G} a R^{G}\right) R\right]_{R} \leq^{\text {ess }} Q(R)_{R} .
$$

We claim that $R a R_{R} \leq^{\text {ess }} \tilde{e} R_{R}$.

First note that $R^{G} a R^{G} R \subseteq \tilde{e} R_{R}$. For the claim, it is enough to show that $R^{G} a R^{G} R_{R} \leq^{\text {ess }} \tilde{e} R_{R}$. Take

$0 \neq \tilde{e} r \in \tilde{e} R$ with $r \in R$. Then there exists $r_{1} \in R$ such that $0 \neq \tilde{e} r r_{1} \in R^{G} a R^{G} R+r_{R^{G}}\left(R^{G} a R^{G}\right) R$. Say err $r_{1}=x+y$, where $x \in R^{G} a R^{G} R$ and $y \in r_{R^{G}}\left(R^{G} a R^{G}\right) R$.

Then $\tilde{e} r r_{1}=\tilde{e} \tilde{e} r r_{1}=\tilde{e} x+\tilde{e} y$.

Put $y=\sum p_{i} s_{i}$ with $p_{i} \in r_{R^{G}}\left(R^{G} a R^{G}\right)$ and $s_{i} \in R$.

Then $\tilde{e} y=\sum \tilde{e} p_{i} s_{i}$. In this case, $\tilde{e} p_{i}=0$ for all $i$. To see this, assume on the contrary that there is $p_{i}$ such that $\tilde{e} p_{i} \neq 0$. Note that $0 \neq \tilde{e} p_{i} \in \tilde{e} R^{G}$.

Thus there exists $r_{0} \in R^{G}$ such that $0 \neq \tilde{e} p_{i} r_{0} \in R^{G} a R^{G}$ because $R^{G} a R_{R^{G}}^{G} \leq^{\text {ess }} \tilde{e} R_{R^{G}}^{G}$.

Also $\tilde{e} p_{i} r_{0} R^{G} a R^{G} \subseteq \tilde{e} p_{i} R^{G} a R^{G}=\tilde{e} 0=0$ because $p_{i} \in r_{R^{G}}\left(R^{G} a R^{G}\right)$. Therefore we have that $0 \neq \tilde{e} p_{i} r_{0} \in$ $R^{G} a R^{G} \cap r_{R^{G}}\left(R^{G} a R^{G}\right)=0$, a contradiction. Thus $\tilde{e} p_{i}=0$ for all $i$, so $\tilde{e} y=0$. Hence $\tilde{e} r r_{1}=\tilde{e} x+\tilde{e} y=\tilde{e} x$. Now since $x \in R^{G} a R^{G} R$, write $x \in \sum t_{i} z_{i}$ with $t_{i} \in R^{G} a R^{G}$ and $z_{i} \in R$. Then $\tilde{e} x=\sum \tilde{e} t_{i} z_{i}=\sum t_{i} z_{i}=x$ because $t_{i} \in R^{G} a R^{G} \subseteq \tilde{e} R^{G}$. So $0 \neq \tilde{e} r r_{1}=\tilde{e} x=x \in R^{G} a R^{G} R$. Therefore $R a R_{R} \leq^{\text {ess }} \tilde{e} R_{R}$. Note that

$$
\begin{aligned}
\operatorname{Cen}\left(Q\left(R^{G}\right)\right) & =[\operatorname{Cen}(Q(R))]^{G} \text { by Lemma 5. Therefore } \\
\tilde{e} & \in B\left(Q\left(R^{G}\right)\right) \subseteq \operatorname{Cen}\left(Q\left(R^{G}\right)\right) \\
& =[\operatorname{Cen}(Q(R))]^{G} \subseteq \operatorname{Cen}(Q(R))
\end{aligned}
$$

Hence $R a R=R R^{G} a R^{G} R \subseteq R \tilde{e} R^{G} R=\tilde{e} R$.

As $R a R$ is a $G$-invariant two-sided ideal of $R$ and $R$ is $R$-p.q.-Baer, there is $f \in S_{l}(R)$ such that

$r_{R}(R a R)=f R$. From [9], $f \in B(R)$. As $\tilde{e} \in B(Q(R))$ and $R a R_{R} \leq^{\text {ess }} \tilde{e} R_{R}$, it follows that $\tilde{e}=1-f \in R$ by Lemma 6, so $\tilde{e} \in R^{G}$.

Therefore $e R^{G}=\tilde{e} R^{G} \subseteq R^{G}$, and thus $e \in R^{G}$.

So $B_{p}\left(Q\left(R^{G}\right)\right) \subseteq R^{G}$, and hence $R^{G}$ is p.q.-Baer by Lemma 9.

2) We recall that a reduced p.q.-Baer ring is Rickart. Thus if $R$ is reduced $G$-p.q.-Baer, then $R^{G}$ is Rickart from 1).

\section{Conclusion}

In [14], the quasi-Baer property of fixed rings under finite group actions on a semiprime ring and their applications to $C^{*}$-algebras have been studied (see also $[17,18]$ ). Motivated by investigations in [14], in this paper we investigate the right p.q.-Baer property of fixed rings under finite group actions on a given semiprime ring. Assume that $R$ is a semiprime ring with a finite group $G$ of $\mathrm{X}$-outer ring automorphisms of $R$. Then we show that if $R$ is $G$-p.q.-Baer, then $R^{G}$ is p.q.-Baer. Thus if $R$ is a semiprime p.q.-Baer ring with finite group $G$ of X-outer ring automorphisms of $R$, then $R^{G}$ is p.q.-Baer. 


\section{REFERENCES}

[1] G. F. Birkenmeier, J. Y. Kim and J. K. Park, "Principally Quasi-Baer Rings,” Communications in Algebra, Vol. 29, No. 2, 2001, pp. 639-660. doi:10.1081/AGB-100001530

[2] G. F. Birkenmeier, H. E. Heatherly, J. Y. Kim and J. K. Park, "Triangular Matrix Representations," Journal of Algebra, Vol. 230, No. 2, 2000, pp. 558-595. doi:10.1006/jabr.2000.8328

[3] G. F. Birkenmeier, J. Y. Kim and J. K. Park, “Quasi-Baer Ring Extensions and Biregular Rings," Bulletin of the Australian Mathematical Society, Vol. 61, No. 1, 2000, pp. 39-52. doi:10.1017/S0004972700022000

[4] G. F. Birkenmeier, J. Y. Kim and J. K. Park, "A Sheaf Representation of Quasi-Baer Rings,” Journal of Pure and Applied Algebra, Vol. 146, No. 3, 2000, pp. 209-223. doi:10.1016/S0022-4049(99)00164-4

[5] G. F. Birkenmeier and J. K. Park, "Triangular Matrix Representations of Ring Extensions,” Journal of Algebra, Vol. 265, No. 2, 2003, pp. 457-477. doi:10.1016/S0021-8693(03)00155-8

[6] G. F. Birkenmeier, J. Y. Kim and J. K. Park, "Polynomial Extensions of Baer and Quasi-Baer Rings," Journal of Pure and Applied Algebra, Vol. 159, No. 1, 2001, pp. 2542. doi:10.1016/S0022-4049(00)00055-4

[7] W. E. Clark, "Twisted Matrix Units Semigroup Algebras,” Duke Mathematical Journal, Vol. 34, No. 3, 1967, pp. 417-423. doi:10.1215/S0012-7094-67-03446-1

[8] A. Pollingher and A. Zaks, "On Baer and Quasi-Baer Rings,” Duke Mathematical Journal, Vol. 37, No. 1, 1970, pp. 127-138. doi:10.1215/S0012-7094-70-03718-X

[9] G. F. Birkenmeier, "Idempotents and Completely Semi- prime Ideals," Communications in Algebra, Vol. 11, No. 6, 1983, pp. 567-580. doi:10.1080/00927878308822865

[10] T. Y. Lam, "Lectures on Modules and Rings," Springer, Berlin, 1998.

[11] J. W. Fisher and S. Montgomery, "Semiprime Skew Group Rings,” Journal of Algebra, Vol. 52, No. 1, 1978, pp. 241-247. doi:10.1016/0021-8693(78)90272-7

[12] M. Cohen, "Morita Context Related to Finite Automorphism Groups of Rings,” Pacific Journal of Mathematics, Vol. 98, No. 1, 1982, pp. 37-54.

[13] S. Montgomery, "Outer Automorphisms of Semi-Prime Rings,” Journal London Mathematical Society, Vol. 18, No. 2, 1978, pp. 209-220. doi:10.1112/jlms/s2-18.2.209

[14] H. L. Jin, J. Doh and J. K. Park, "Group Actions on Quasi-Baer Rings,” Canadian Mathematical Bulletin, Vol. 52, 2009, pp. 564-582. doi:10.4153/CMB-2009-057-6

[15] J. Osterburg and J. K. Park, "Morita Contexts and Quotient Rings of Fixed Rings," Houston Journal of Mathematics, Vol. 10, 1984, pp. 75-80.

[16] G. F. Birkenmeier, J. K. Park and S. T. Rizvi, "Principally Quasi-Baer Ring Hulls,” Advances in Ring Theory, 2010, pp. 47-61. doi:10.1007/978-3-0346-0286-0 4

[17] G. F. Birkenmeier, J. K. Park and S. T. Rizvi, "Hulls of Semiprime Rings with Applications to C*-Algebras," Journal of Algebra, Vol. 322, No. 2, 2009, pp. 327-352. doi:10.1016/j.jalgebra.2009.03.036

[18] G. F. Birkenmeier, J. K. Park and S. T. Rizvi, "The Structure of Rings of Quotients,” Journal of Algebra, Vol. 321, No. 9, 2009, pp. 2545-2566. doi:10.1016/j.jalgebra.2009.02.013 